Proc. Indian Acad. Sci. (Earth Planet. Sci.), Vol. 102, No. 1, March 1993, pp. 49-72.

(C) Printed in India.

\title{
Mechanisms of variability and predictability of the tropical coupled ocean-atmosphere system
}

\author{
B N GOSWAMI ${ }^{\dagger}$, SUDHA SELVARAJAN ${ }^{\dagger}$ and V KRISHNAMURTHY* \\ ${ }^{\dagger}$ Centre for Atmospheric Sciences, Indian Institute of Science, Bangalore 560012, India \\ *International Institute for Earth, Environmental and Marine Sciences and Technologies, \\ 34014 Miramare, Trieste, Italy
}

\begin{abstract}
A conceptual model is proposed to explain the observed aperiodicity in the short term climate fluctuations of the tropical coupled ocean-atmosphere system. This is based on the evidence presented here that the tropical coupled ocean-atmosphere system sustains a low frequency inter-annual mode and a host of higher frequency intra-seasonal unstable modes. At long wavelengths, the low frequency mode is dominant while at short wavelengths, the high frequency modes are dominant resulting in the co-existence of a long wave low frequency mode with some short wave intra-seasonal modes in the tropical coupled system. It is argued that due to its long wavelength, the low frequency mode would behave like a linear oscillator while the higher frequency short wave modes would be nonlinear. The conceptual model envisages that an interaction between the low frequency linear oscillator and the high frequency nonlinear oscillations results in the observed aperiodicity of the tropical coupled system. This is illustrated by representing the higher frequency intra-seasonal oscillations by a nonlinear low order model which is then coupled to a linear oscillator with a periodicity of four years. The physical mechanism resulting in the aperiodicity in the low frequency oscillations and implications of these results on the predictability of the coupled system are discussed.
\end{abstract}

Keywords. Aperiodicity; inter-annual variability; tropical coupled ocean-atmosphere system; predictability; convergence feedback; multiple periodic attractors; low frequency oscillator; non linear intra-seasonal modes.

\section{Introduction}

The short term climate in the tropics is dominated by the El Nino and Southern Oscillation (ENSO), an irregularly fluctuating inter-annual phenomenon. These fluctuations are associated with large scale climatic anomalies such as devastating droughts in western Pacific, torrential floods over eastern tropical Pacific and damaging weather patterns over other parts of the world such as north America. Therefore, ability to predict these climatic fluctuations has tremendous socioeconomic impact. During the last decade or so, it has been well established that the tropical atmosphere and the ocean interact strongly in time scales longer than a season and that the ENSO is a result of such interactions between the atmosphere and the ocean (see Philander 1990 for a review).

For time scales in which the atmosphere can be considered in isolation, the predictability of the atmosphere has been studied extensively (e.g. Shukla 1985 for a review). It has been shown that there exists a limit on the predictability of the instantaneous state of the atmosphere. This limit, which happens to be between two to three weeks, depends on the internal dynamics (nonlinearity, instability etc.) and external forcing in the system. While, it may be impossible to predict the day to day 
fluctuations of the weather beyond a few weeks, the averages or climatic conditions of the atmosphere may be more predictable. It has been argued (Charney and Shukla 1981; Shukla 1981) that the mean fields in the tropics may be more predictable compared to those in the extratropics as slowly varying boundary conditions (such as the SST, soil moisture, vegetation cover etc.) introduce a slowly varying component to the forcing of the tropical atmosphere. Thus, a conceptual basis for tropical climate
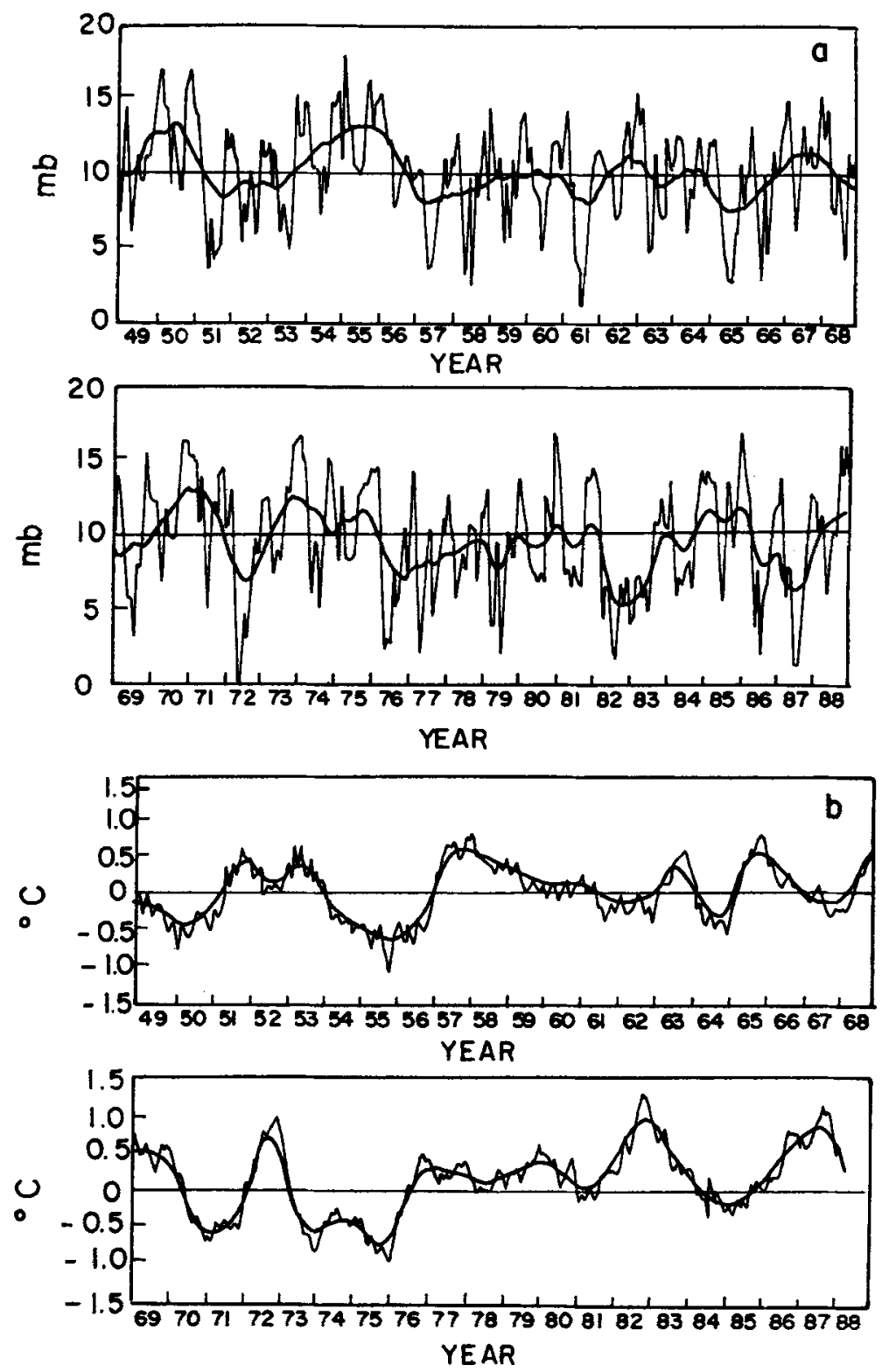

Figure 1. Monthly mean time series of (a) a classical index of Southern Oscillation i.e. the pressure difference between Easter Island and Darwin in $\mathrm{mb}$, (b) sea surface temperature anomalies in the eastern equatorial Pacific region $T_{s}^{\mathrm{EEP}}\left(20^{\circ} \mathrm{S}-20^{\circ} \mathrm{N}, 80 \mathrm{~W}-180 \mathrm{~W}\right)$ in ${ }^{\circ} \mathrm{C}$. The thick line in (a) represents a 12-month running mean and in (b) the thick line is a 15-month weighted average. 
predictability is established. However, as soon as we go to time scales longer than a season, we cannot consider the atmosphere in isolation. In these time scales the tropical atmosphere and the ocean interact strongly. Therefore, to address the question of predictability of the tropical climate, we must consider the tropical coupled oceanatmosphere system. The sea surface temperatures (SST) modulate the atmospheric heating which drives the surface winds which in turn drive the oceanic currents and thereby affects the SST distribution. Hence the concept of slowly varying boundary forcing will not be applicable to the coupled system. The only external forcing to the coupled system is the annually varying solar forcing. Therefore, the predictability of the coupled system will be mainly governed by internal dynamics. The internal dynamics encompasses the nature of the nonlinearities, the dominant natural modes of oscillations and the instabilities of the system and their saturation mechanisms. As opposed to the atmosphere alone, however, the internal dynamics itself may lead to slow modes of oscillation of the coupled system leading to long range predictability of the system.

To get an idea of the nature of internal dynamics of the tropical coupled system, it is illustrative to examine some features of inter-annual variability in the tropics. Figure 1 shows the time series of one index of the Southern Oscillation (SO) and another index of the El Nino (EN). Several features are noteworthy. High frequency intra-seasonal oscillations (light curve) are superimposed on a dominant low frequency oscillation (heavy curve) of both the atmosphere and the ocean. There is a very high coherence between the atmospheric low frequency oscillations and the oceanic low frequency oscillations. The high frequency fluctuations of the two systems are not well correlated. The low frequency oscillation is quite irregular but seems to have a preferred periodicity of about four years. This is seen in figure 2 which shows power spectra of similar SO and EN indices. It is clear that there is a dominant peak around 4 years but it is a broad band spectrum, indicating the already noted aperiodicity. If the inter-annual variability were perfectly periodic, it would be infinitely predictable. The existence of a dominant natural slow mode of oscillation indicates the existence of some long range predictability. However, the aperiodicity is going to introduce a limit on this predictability.

While there exists a large volume of studies on the predictability of the atmosphere (see Shukla 1985 for more references), only a few studies (Goswami and Shukla 1991; Blumenthal 1991; Latif and Flugel 1991) have attempted to make a quantitative estimate of the predictability of the coupled system so far. Goswami and Shukla (1991) follow the classical method of studying predictability and conducted a large ensemble of identical twin experiments with the Cane and Zebiak (1985; Zebiak and Cane 1987) model and showed that the growth of small errors in the coupled system is governed by two time scales. The fast time scale has an error doubling time of about 5 months, while the slow time scale has an error doubling time of about 15 months. Blumenthal (1991) following a different approach arrived at the same two time scales. Latif and Flugel (1991) also obtained the fast time scale using a more complex ocean model but their forecasts were not long enough to estimate the slow time scale. The two time scales first discovered by Goswami and Shukla (1991) seems to be a generic feature of the coupled system. The slow time scale which provides a basis for long range predictability appears to arise as a result of the dominant four year cycle of the system while the fast time scale appears to arise due to the aperiodicity of the system. We are now beginning to understand the physical mechanism for the 4 year cycle. One plausible scenario that seems to explain results of several simple 


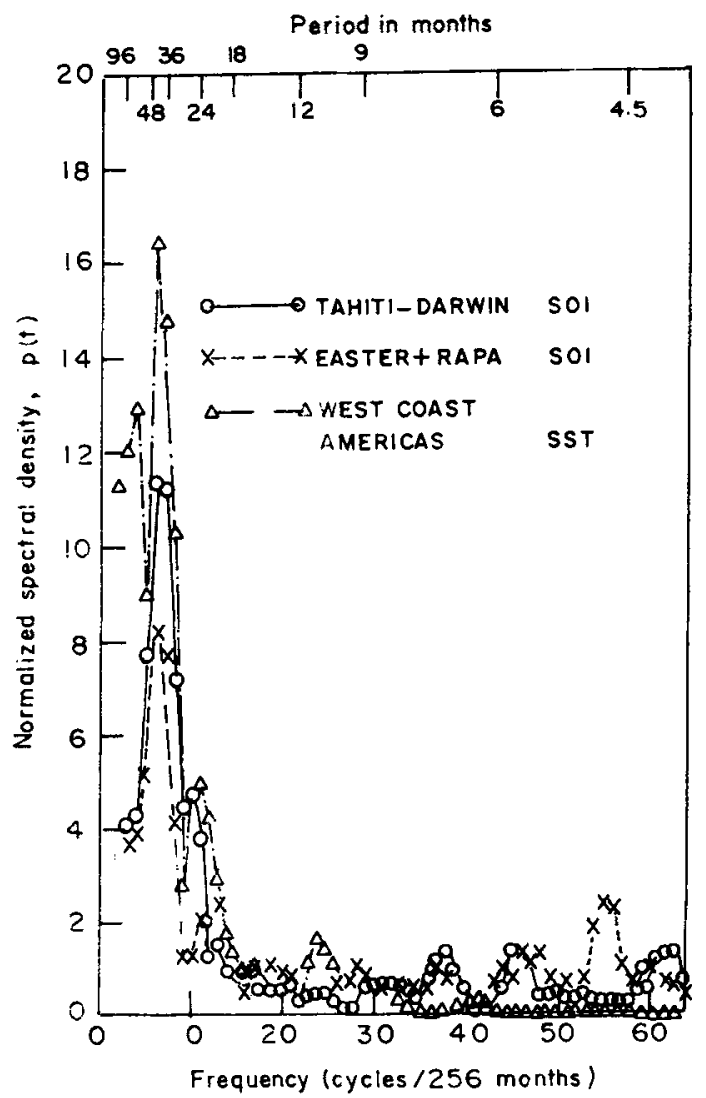

Figure 2. Spectra for mean monthly anomaly time series (September 1953-December 1974) for Tahiti minus Darwin normalized surface pressure difference $(T-D)_{N}$, Easter Island plus Rapa surface pressure $(E+R)$ and SST anomalies near upper Peru coast (4-12 ${ }^{\circ}$ ) (after Rasmusson and Carpenter 1982).

models (Zebiak and Cane 1987; Battisti 1988; Schopf and Suarez 1988) as well as some complex coupled GCM simulations (Philander et al 1992) is the delayed oscillator mechanism (Suarez and Schopf 1988; Battisti and Hirst 1989). The delayed oscillator mechanism has its root in the fact that the coupled ocean-atmosphere system in the equatorial Pacific is capable of sustaining a low frequency unstable mode (Philander et al 1984; Hirst 1986). The positive feedback associated with this unstable air-sea interaction and the negative feedback associated with the Rossby waves reflected from the western boundary as Kelvin waves leads to an oscillating mode with a period around 4 years. However, no such conceptual picture is available for explaining the aperiodicity of the system. The objective of this article is to attempt to identify important physical process(es) responsible for the aperiodicity of the low frequency variability in the coupled system. Then, we shall present a conceptual model of the nonlinear interactions leading to this aperiodicity.

In general aperiodicity may be attributed to nonlinear interaction between more than one mode (or degrees of freedom) of the system. Early instability analyses (Philander et al 1984; Hirst 1986, 1988) showed the existence of only one unstable low frequency oscillation which has been invoked in explaining the four year 
oscillation. If there exists only one low frequency oscillatory mode of the system, it is unlikely to produce aperiodicity by itself. The task, therefore, is to identify other unstable modes that are capable of coexisting in the system. In an attempt to identify the physical process that may be responsible for introducing new unstable modes in the coupled system, we carried out a series of sensitivity studies with the Cane-Zebiak model. These results are presented in section 2 . These sensitivity experiments indicate that "convergence feedback" or wave-CISK mechanism included in the parameterization of atmospheric heating may introduce some new unstable modes. To establish this, we carried out a stability analysis of the linear coupled system including the effect of wave-CISK. These results are presented in section 3. It shows that the convergence feedback indeed introduces a set of new higher frequency unstable modes. In section 4 we present a paradigm for generating the aperiodicity in the low frequency variability of the coupled system and illustrate it with a simple low order dynamical model.

\section{Aperiodicity in the Cane-Zebiak coupled model}

In an attempt to identify physical processes that may be crucial in introducing the aperiodicity in the inter-annual variability, a series of sensitivity studies were carried out with the coupled model developed by Cane and Zebiak 1985 (Zebiak and Cane 1987, hereafter referred to as CZ model). This is a simple coupled anomaly model that is successful in simulating both the preferred periodicity of about 4 years and the irregular interval between warm (or cold) events. The ocean in the $\mathrm{CZ}$ model evolves according to linear reduced gravity equations and has a frictionally driven, constant depth, upper mixed layer. The atmospheric model dynamics is that of Gill (1980). The seasonal cycle is included through prescribed climatological surface winds, sea surface temperature (SST) and ocean currents. The SST anomalies (SSTA) are predicted through a fully nonlinear thermodynamic equation. The atmosphere is coupled to the ocean through the surface wind stress parameterized by a drag law. The ocean is coupled to the atmosphere through the parameterization of atmospheric heating which has two parts. The first part is proportional to SSTA and depends nonlinearly on the mean SST. The second part is proportional to low level convergence anomaly but operates only when the total flow is convergent. In this way, the coupling depends on the annual cycles of mean convergence and mean SST. The model has several adjustable parameters such as the oceanic equivalent depth, the sharpness and amplitude of the mean thermocline, the strength of atmospheric heating proportional to the SSTA, the strength of the atmospheric heating proportional to the convergence anomaly ("convergence feedback") and atmospheric dissipation. For a range of parameters, the model simulates several features of the observed ENSO variability quite well (figure 3a). This model has shown considerable skill in making hindcast for past ENSO events (Cane et al 1986; Barnett et al 1988). We shall call the set of parameters used by Zebiak and Cane (1987) as the "standard set". Battisti and Hirst (1989) showed that the dominant oscillation with periodicity of about 4 years arises in the $\mathrm{CZ}$ model due to a delayed oscillator mechanism. The aperiodicity in the model arises due to nonlinearities in the model. The model has explicit advective nonlinearities in the thermodynamic equation and implicit nonlinearities in the coupling processes. The dominant quasi four year cycle arises due to the dominant low frequency mode of the coupled system found by Hirst (1986) 

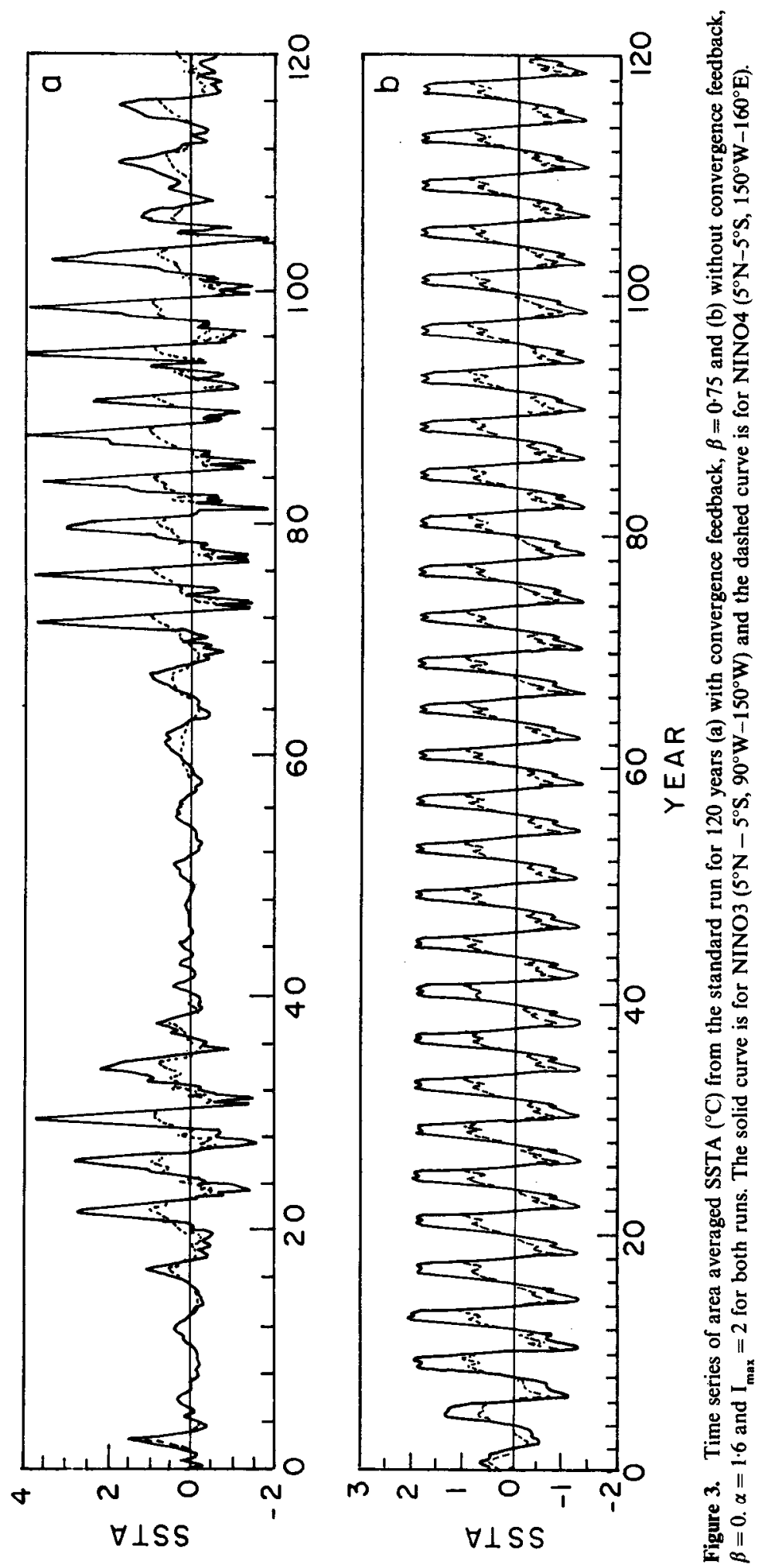

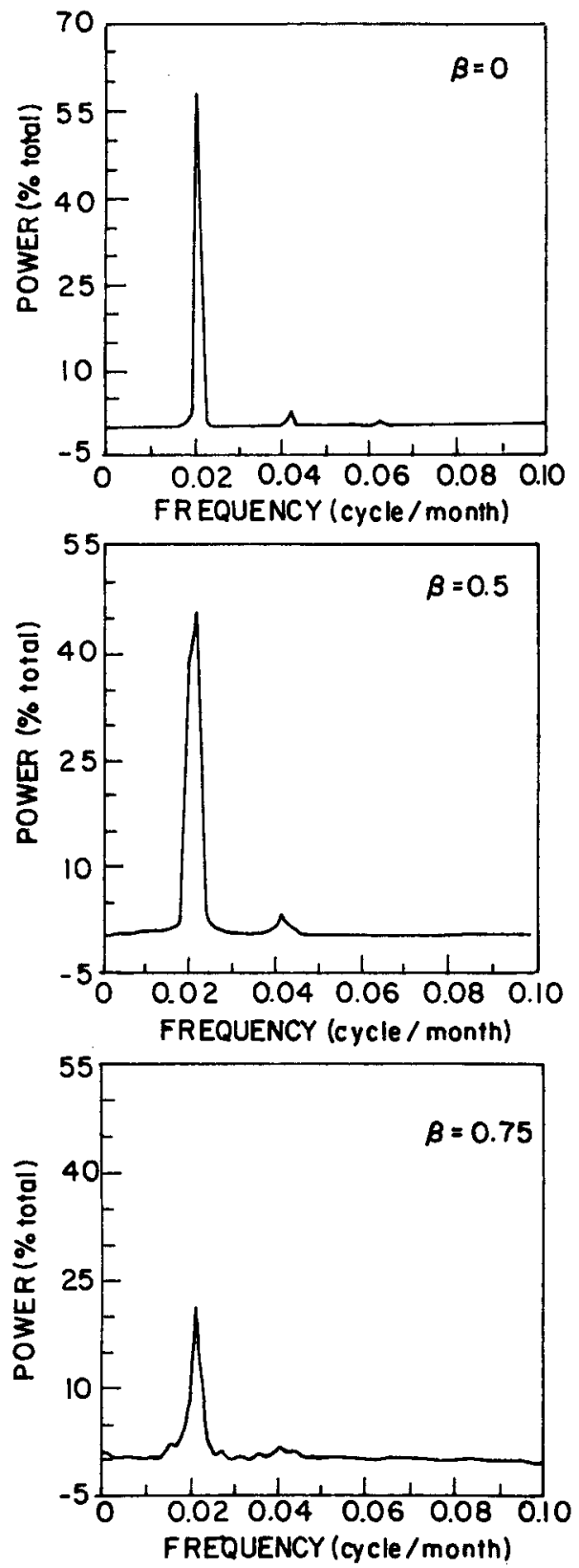

Figure 4. Power spectra of NINO3 SSTA from three simulations with three different values of the strength of the convergence feedback $(\beta)$. Power is shown as percentage of the total and unit of frequency is cycles per month (from Goswami and Shukla 1993).

and others. In order that the spectrum around quasi four year period be broad, there must be more than one unstable mode. However, Hirst (1986) and other early stability analyses indicate the existence of only one low frequency mode. In these studies, the atmospheric heating anomaly was assumed to be directly proportional to the SST anomalies. In addition to this process, the $\mathrm{CZ}$ model also contains the 
convergence feedback process. We argued that inclusion of physical processes not taken into account in earlier stability analyses may introduce other unstable modes in the coupled system. To test this possibility, we carried out some sensitivity experiments with the coupling processes of the $\mathrm{CZ}$ model (Goswami and Shukla 1993).

It is found that for the standard set of parameters, the aperiodicity of the CZ model may be related to the "convergence feedback" effect. For example, if the convergence feedback is set to zero (figure $3 \mathrm{~b}$ ), the model variability becomes periodic. Figure 4 shows the power spectra of one index of model variability for three different strengths of the convergence feedback. It is seen that for $\beta=0$, the spectrum is a line spectrum with period of about 4 years and as $\beta$ is increased the spectrum broadens around this periodicity. As parameterized in this model, the "convergence feedback" is proportional to convergence anomaly but operates only when the total flow is convergent. Convergence anomalies, occur due to the SST anomalies and are nearly colocated. The SST anomalies in the model are largest in the eastern Pacific. Therefore, when the SSTA is large in the eastern Pacific e.g. in the warm phases of ENSO, if the mean convergence field happens to be around the equator or south of the equator, it overlies the SST anomalies and the convergence feedback has the highest chance
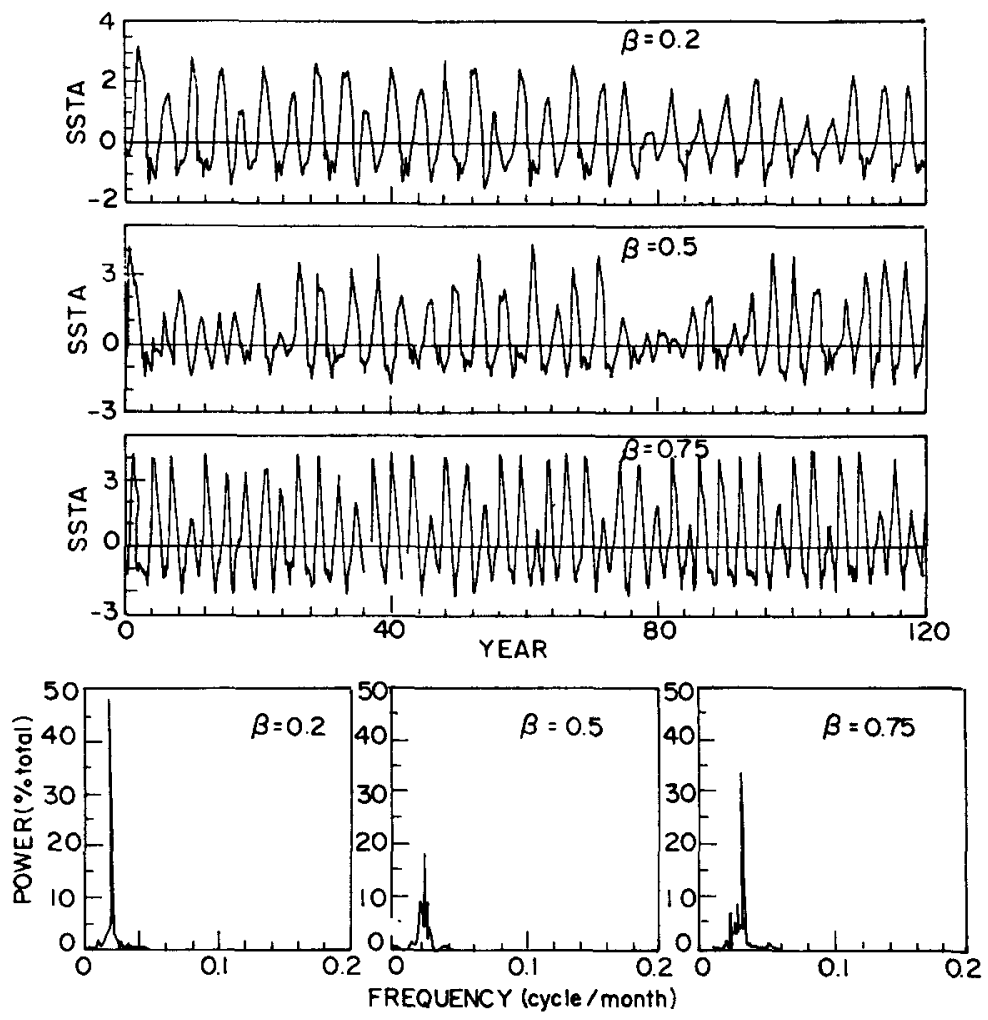

Figure 5. Time evolution of NINO3 SSTA $\left({ }^{\circ} \mathrm{C}\right)$ corresponding to three different strengths of the convergence feedback (top three panels) when the mean convergence field is kept fixed at March values. The other mean fields have normal annual cycle. The power spectra of the three time series are also shown below (from Goswami and Shukla 1993). 
of enhancing the atmospheric heating. On other occasions, when the mean convergence field and SSTA field are not colocated, the feedback may not contribute to the atmospheric heating field. An examination of the annual March of the mean convergence field reveals that the mean convergence field is located around the equator during February-April and farthest from the equator during September. Therefore, we expect that the "convergence feedback" would be most effective during March and least effective some time around September. Figures 5 and 6 show that, indeed that is what happens in the $\mathrm{CZ}$ model. When the mean convergence field is held fixed at March values, even a small strength of the convergence feedback is able to make the model variability aperiodic. This indicates that during March, the efficiency of the convergence feedback process is enhanced. On the other hand, if the mean convergence field is held fixed at September values, the model variability remains quasi periodic even for a very large strength of the convergence feedback.

These sensitivity studies with the $\mathrm{CZ}$ model indicate that the convergence feedback introduces aperiodicity in the standard version of the $\mathrm{CZ}$ model. At this point, we postulate that the convergence feedback introduces new unstable modes in the coupled system and an interaction between these new modes and the low frequency mode results in aperiodicity of the low frequency mode. In the next section, we examine theoretically whether the convergence feedback is indeed capable of introducing some new unstable coupled modes.
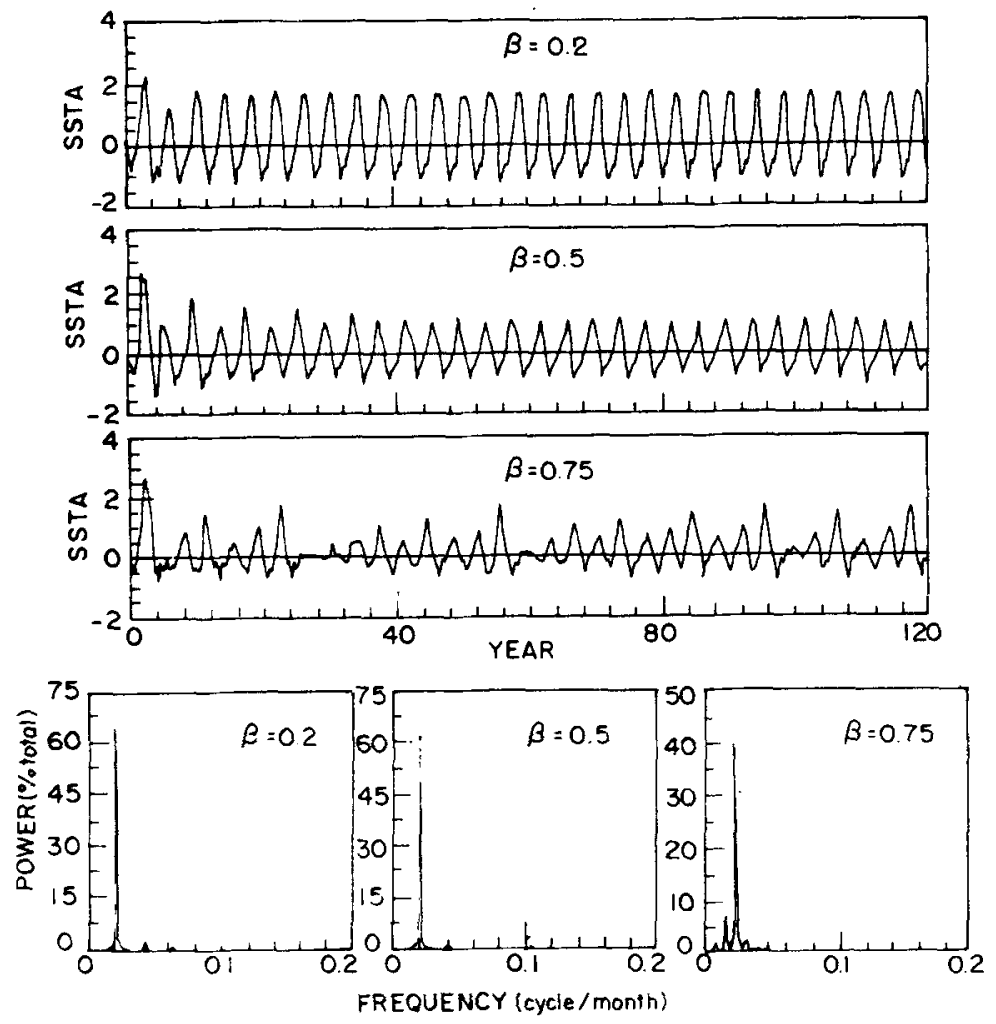

Figure 6. Same as figure 5 except when the mean convergence field is held fixed at September values (from Goswami and Shukla 1993). 


\section{Linear stability analysis of the coupled system with convergence feedback}

The stability analyses of the linear coupled ocean-atmosphere system have been instrumental in identifying natural coupled unstable oscillations. These studies provide a basis to understand the nature of the observed low frequency variability in the tropics. Early such analyses (Philander et al 1984; Hirst 1986, 1988) showed that although the linear tropical atmosphere and ocean by themselves may be stable, the coupling between the two may drive the coupled system unstable. All these studies showed that the coupled system is capable of sustaining one low frequency unstable inter-annual mode. The nature of this mode depends on the nature of the thermodynamics that determines the evolution of the sea surface temperature anomalies (Hirst 1986). Equations governing the evolution of the total energy of the atmospheric and the oceanic perturbations may be derived from the linear coupled equations. They show that the perturbations of the coupled system may grow if convergent atmospheric low level winds overlie convergent currents in the upper ocean. Since the atmospheric winds result from atmospheric heating, it is reasonable to think that the atmospheric heating distribution dictates which mode may be unstable. The atmospheric heating distribution in turn depends on the SST distribution governed by the ocean thermodynamics. The stability analyses (referred to earlier) assumed atmospheric heating to be directly proportional to SSTA. This rather simple minded parameterization of the atmospheric heating field assumes that the tropical ocean is warm and an increase in SST enhances evaporation which is immediately converted to latent heating. However, on time scales of a month or longer, the local evaporation over a large precipitating region accounts for only about one third of the precipitation (Shukla and Wallace 1983). The rest comes from low level convergence. An initial organized heating in the atmosphere results in low level convergence. Convergence of moisture associated with the enhanced low level convergence further intensifies the heating. This process, which we refer to as "convergence feedback" can influence the air-sea interaction in the following ways. The convergence feedback essentially reduces the effective static stability of the system (Lau and Shen 1988; Hirst and Lau 1990) thereby reducing the phase speed of the atmospheric waves. With the phase speed of the atmospheric waves sufficiently reduced, the speed of some of the atmospheric waves may become comparable to that of some oceanic waves. This introduces the possibility of some atmospheric modes being strongly coupled to some oceanic modes. Lau and Shen (1988) studied the role of convergence feedback on coupling between an atmospheric Kelvin wave and an oceanic Kelvin wave. This study excluded the possibility that heating associated with one oceanic mode may generate a number of other atmospheric modes. Hirst and Lau (1990) extended the study of Lau and Shen (1988) to more general equatorial beta plane system but still restricted their analysis only to "advective" and "upwelling" limits of oceanic thermodynamics. Although, these special cases are useful in understanding the dynamics of the coupled system, the general ocean thermodynamic case (model III of Hirst 1986) is fundamentally different and may be more relevant to the real system. Recently, we carried out stability analyses of a coupled system with linear first baroclinic mode atmosphere and linear reduced gravity ocean with general oceanic thermodynamics involving both advection and upwelling (Goswami and Selvarajan 1991; Selvarajan and Goswami 1992). The atmospheric heating was proportional to SSTA as well as the wave convergences. 


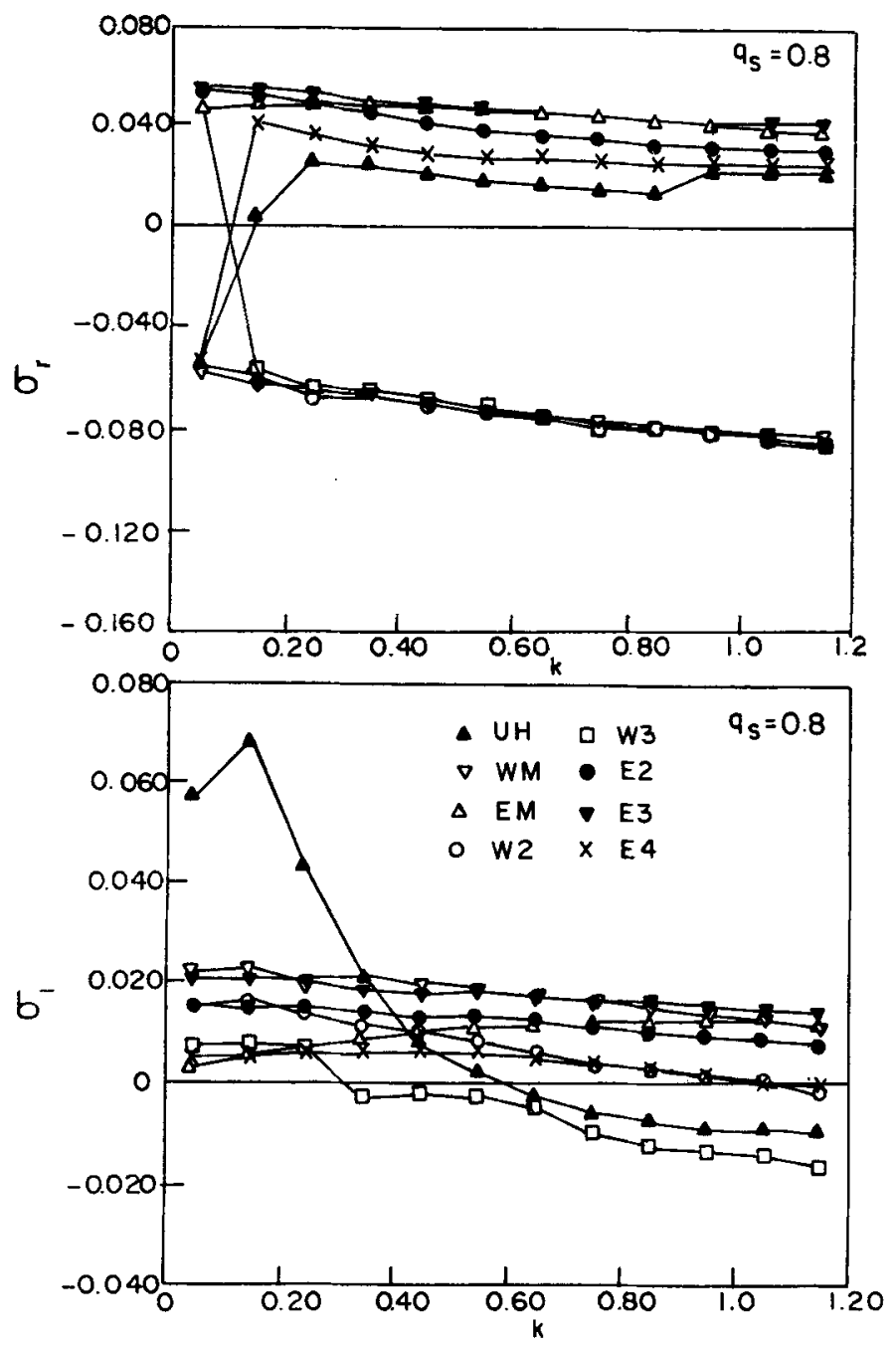

Figure 7. Nondimensional dispersion relation (all modes) for the strength of convergence feedback, $q_{s}=0.8$. This represents 80 percent reduction of dry static stability. $\sigma_{r}, \sigma_{i}$, and $k$ are the nondimensional real, imaginary frequencies and wavenumber respectively. UH, WM and EM represent the unstable low frequency mode (Hirst's mode) and maximally growing westward and eastward propagating modes. The other symbols refer to other eastward propagating $(E)$ and westward propagating $(W)$ intra-seasonal modes. (From Selvarajan and Goswami 1992).

Details about the basic equations, parameterization of heating and method of solution may be found in Selvarajan and Goswami (1992).

We found that the convergence feedback not only significantly modifies the low frequency unstable mode found by Hirst (1986), but also introduces several new higher frequency intra-seasonal unstable modes if the strength of the convergence feedback exceeds a critical limit. Figure 7 shows the dispersion relation for the unstable modes for a representative strength of the convergence feedback $\left(q_{s}\right)$. The low frequency mode found by Hirst (1986) is marked UH. The convergence feedback introduces 

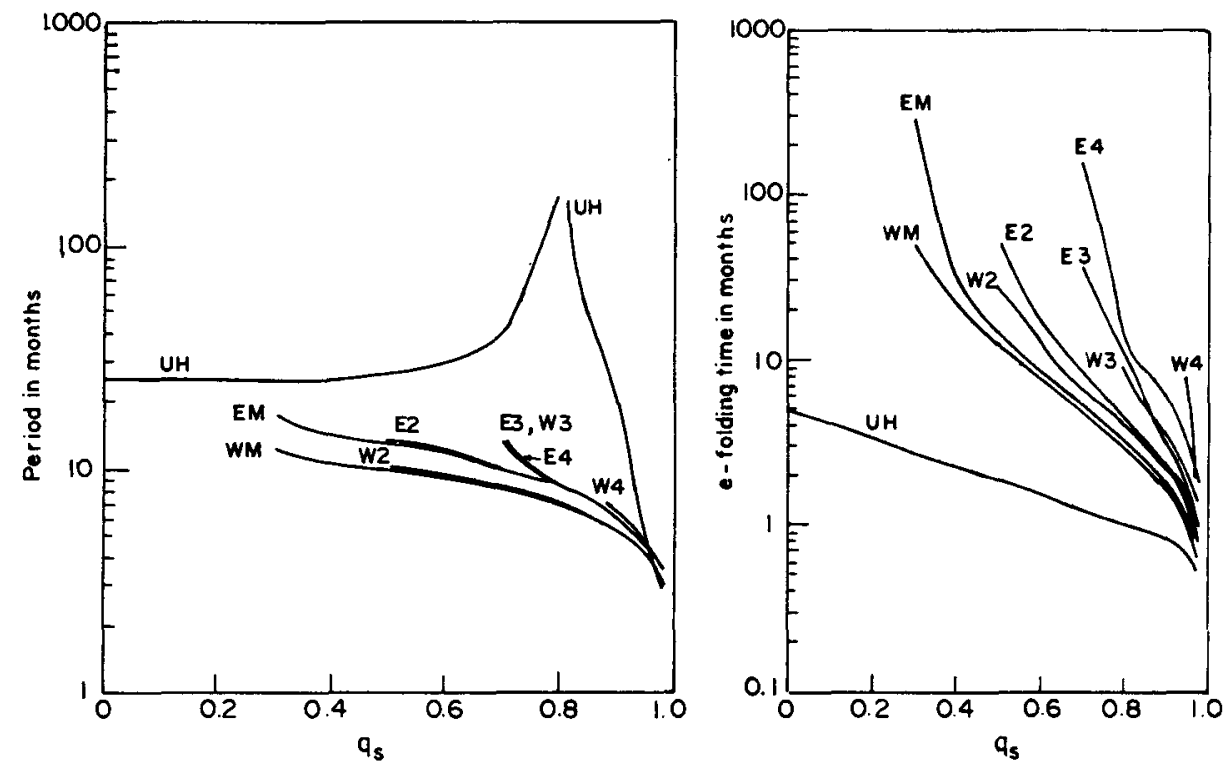

Figure 8. Dependence of the period and e-folding time of all the unstable modes on the strength of convergence feedback $\left(q_{s}\right)$ for nondimensional wavenumber, $k=0.15$ (wavelength of about $10,000 \mathrm{~km}$ ). The symbols have the same meaning as in figure 7. (From Selvarajan and Goswami 1992)

some new unstable intra-seasonal modes. The growth rate of the new unstable modes decreases very slowly with wave number. We note that at long wavelengths the UH mode still dominates. At shorter wavelengths, while the UH mode is stable, the new modes are still unstable. Thus, it is likely that some short wave higher frequency modes will coexist with the longwave low frequency mode. The growth rates of the low frequency UH mode as well as that of the new intra-seasonal modes depend sensitively on the strength of the convergence feedback (figure 8). We also note from figure 8 that for sufficiently high strength of convergence feedback, both the period and growth rates of the UH mode and those of the new unstable modes become comparable. Further details on the structure and maintenance of the new unstable modes are discussed at length in Goswami and Selvarajan (1991) and Selvarajan and Goswami (1992).

\section{A paradigm for aperiodicity in the coupled system}

The low frequency unstable mode (UH mode) together with the reflections of the Rossby waves from the western boundary is attributed for the dominant oscillation with a period of about four years (Schopf and Suarez 1988 and Battisti and Hirst 1989). Our discovery, discussed in the previous section, indicates that the dominant low frequency oscillation with a period of about four years will coexist with higher frequency intra-seasonal oscillations with shorter wavelengths. With this recognition we propose a conceptual model for the aperiodicity in the tropical coupled system. We note that the low frequency oscillation has planetary scales (length scale of about 
6-7 thousand kilometers similar to the observed ENSO anomaly scales). For such planetary scale phenomena, the nonlinear effects are not expected to be strong as the equatorial Rossby number, $U / B_{0} L^{2}$ (where $U$ is a typical velocity scale, $L$ length scale and $\beta_{0}$ is equatorial gradient of the Coriolis parameter) is quite small. Therefore, we assume that the low frequency oscillation may be considered as a linear oscillator. On the other hand, the higher frequency oscillations have much shorter scales and for them the advective nonlinearity may be important. Thus, the evolution of the coupled system may be represented by an interaction between a low frequency linear oscillator and some high frequency nonlinear waves.

To represent the nonlinear interactions between the high frequency waves, we choose a prototype nonlinear system used by Lorenz $(1984,1990)$ to describe some aspects of general circulation of the atmosphere. Coupling these nonlinear equations to a linear oscillator, the equations for the coupled system may be written as

$$
\begin{aligned}
\dot{X} & =-Y^{2}-Z^{2}-a X+a F, \\
\dot{Y} & =X Y-b X Z-C Y+G+\alpha P, \\
\dot{Z} & =b X Y+X Z-C Z+\alpha Q, \\
\dot{P} & =-\omega Q-\beta Y, \\
\dot{Q} & =\omega P-\beta Z,
\end{aligned}
$$

where $\omega$ is the frequency of the low frequency oscillator with a period of four years, $P$ and $Q$ are amplitudes of the sine and cosine phases of the oscillation and $\alpha$ and $\beta$ are coupling strengths. Equations (1)-(3) represent the high frequency component and (4)-(5) represent the low frequency component. The typical period of oscillation of the high frequency coupled modes is in the intra-seasonal range. In order that the nonlinear system (1)-(3) contain these intrinsic scales of the coupled system, we have rescaled Lorenz's (1984) equations by a factor $C$ as (original variables denoted by prime),

$$
\begin{aligned}
& t=t^{\prime} / C, X=C X^{\prime}, Y=C Y^{\prime}, Z=C Z^{\prime}, a=a^{\prime} C, b=b^{\prime} \\
& F=C F^{\prime} \text { and } G=C^{2} G^{\prime}
\end{aligned}
$$

As Lorenz (1984) discusses, $X$ may be interpreted as a zonally averaged field while $Y$ and $Z$ may be interpreted as amplitudes of two wave components. $F$ and $G$ are forcings. $F$ may be interpreted as external zonally symmetric forcing (eg. solar forcing) while $G$ is the zonally asymmetric forcing (eg. land-ocean contrast). However, we have chosen equations (1)-(3) primarily as a prototype nonlinear system containing the essential nonlinearity of the coupled intra-seasonal modes. This may be considered as a "toy model" to derive some insight regarding the nonlinear interactions in the coupled system. We do not attempt to interpret the variables $X, Y, Z$ with any specific variables of the real system. We have used $C=0.5$. A typical time series of the variable $X$ for 50 years is shown in figure 9 without coupling with the low frequency oscillation. Several features of this time series are noteworthy. Firstly, the system seems to have two preferred regimes of oscillations, one active regime with large amplitude relatively low frequency oscillations and another with small amplitude relatively higher frequency oscillations. During the active regimes, the number of oscillations within a year range between 2 and 5. The smaller number of oscillations in a year occurs 


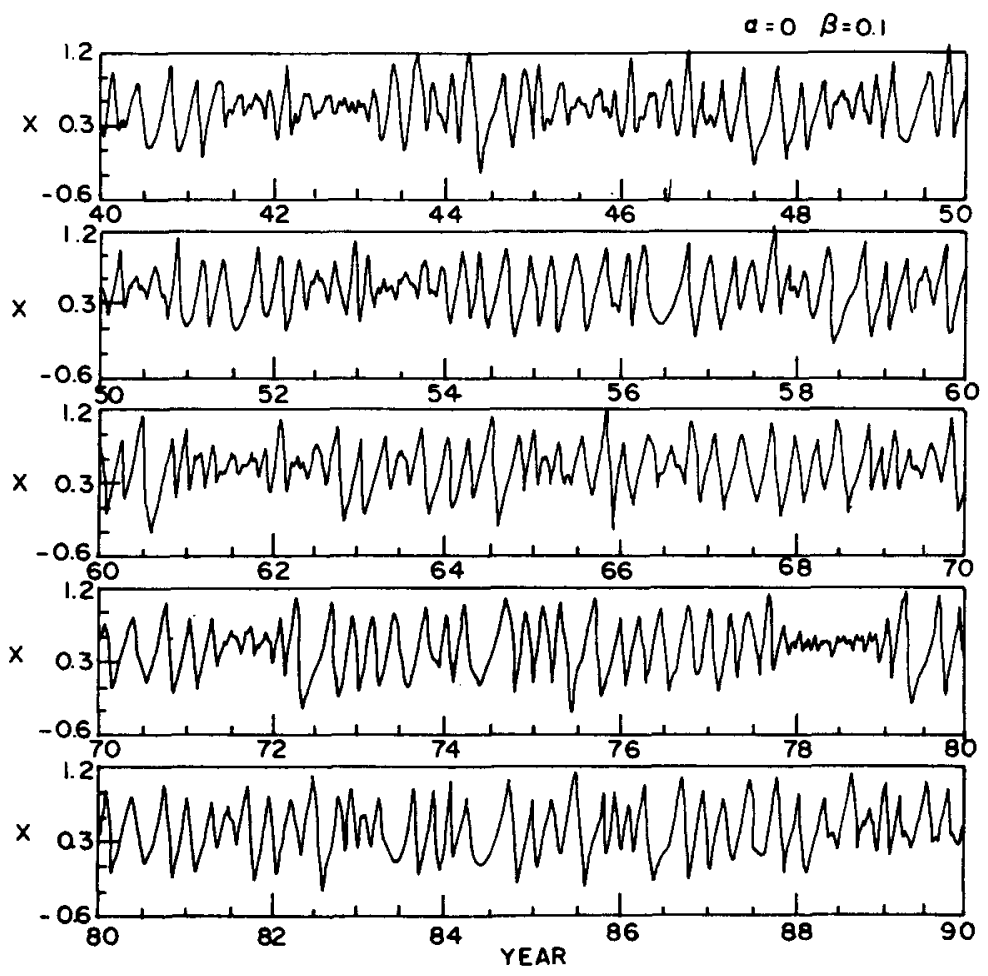

Figure 9. A typical time series of the variable $X$ for 50 years after an initial integration of 40 years for $F_{0}=3 \cdot 5, F_{1}=1 \cdot 0, G_{0}=0.25, \alpha=0 \cdot 0, \beta=0.1$.

(eg. during 56-57, and 84-85 years) when the system tends to reside in a quasi-steady regime. The forcings for this time series has an annual cycle in $F$ and a constant $G$.

The coupling of the high frequency oscillations to the low frequency oscillation is assumed to take place through the nonzonal components $(Y, Z)$. This is based on the following heuristic arguments. As we discussed in the previous section, the amplitude and growth rate of the high frequency oscillations depend crucially on the strength of the convergence feedback (ref. figure 8). The strength of the convergence feedback depends on prevailing mean conditions such as the mean SST distributions. The low frequency oscillation associated with ENSO modulates these mean conditions and generates east-west asymmetry in these conditions. This may be considered as a zonally asymmetric forcing for the high frequency oscillations. This is why we have added the coupling in the $Y$ and $Z$ components of the nonlinear equations. We note that if $\alpha=\beta$, the low order system (1)-(5) conserves total energy $E=\left(X^{2}+Y^{2}+Z^{2}+\right.$ $\left.P^{2}+Q^{2}\right) / 2$, in the absence of forcing and dissipation.

Our objective here is to explore how the high frequency component modifies the low frequency oscillator. Therefore, in figure 10, we show a 240 year time series of $P$ and nine month running mean of $X$ for $\alpha=\beta=0.1$. The time series of $P$ shows that it is quite aperiodic but still has the dominant four year period. This time series has many of the characteristics of the observed time series of ENSO. The low frequency component of $X$, although it has some low frequency variability, does not show any preference for a particular period (this was checked by examining the spectrum of 9 


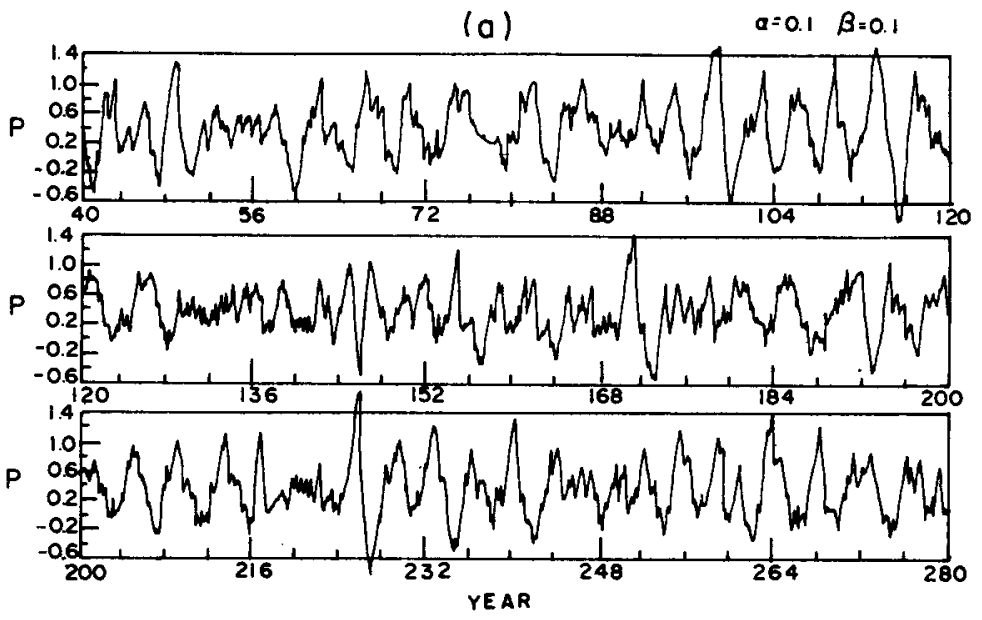

(b)

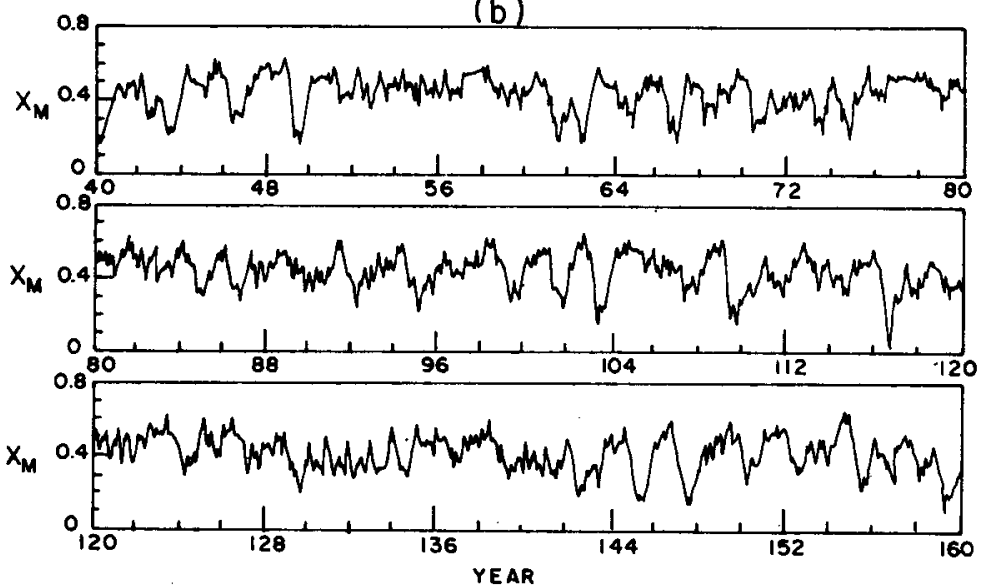

Figure 10. Low frequency variability of the coupled model (a) 240 year time series of $P$. (b) 120 year time series of 9 month running mean of $X . \alpha=0.1, \beta=0.1 . F$ and $G$ are the same as in figure 9.

month running mean of $X$ ). The spectra of $P$ and $X$ for the same case as described in figure 10 are shown in figure $11(\mathrm{a}, \mathrm{b})$. The interaction between the high frequency nonlinear intra-seasonal oscillations and the linear low frequency oscillator results in a broadening of the spectrum of the low frequency oscillations (figure 11a). This is quite similar to the spectrum of observed El Nino or Southern Oscillation indices (ref. figure 2) or that of the spectrum of ENSO indices in coupled models (Zebiak and Cane 1987; Goswami and Shukla 1993). The coupling of the high frequency component to the low frequency component may be looked upon as a slowly varying zonally asymmetric forcing for them. This slow variation in the forcing together with the annual cycle (slowly varying zonally symmetric forcing) results in some low frequency variations in the nonlinear system (figure $11 \mathrm{~b}$ ). The mechanism through which the low frequencies in the nonlinear system are generated may be understood as follows.

To illustrate that the slowly varying forcing associated with the annual cycle of $F=F_{0}+F_{1} \cos 2 \pi t / \tau_{1}$, where $\tau_{1}$ is one year and that associated with $G=G_{0}+$ 

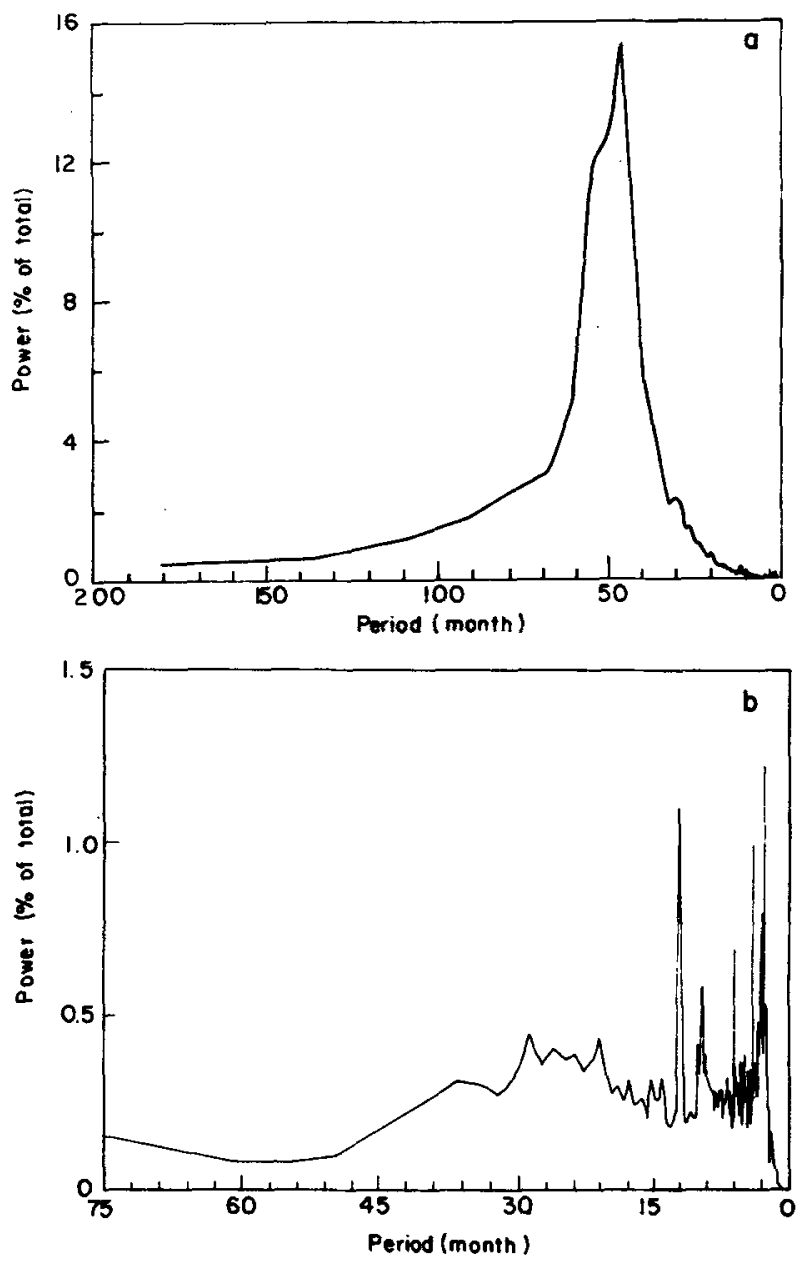

Figure 11. Spectra of (a) $P$ and (b) $X$ for the same parameters as in figure $9 . F$ and $G$ are the same as in figure 9.

$G_{1} \cos 2 \pi t / \tau_{4}$, where $\tau_{4}$ is four years can introduce low frequency variations in the high frequency nonlinear system, we carried out some integrations with $\alpha=\beta=0$ and with $C=1 \cdot 0$. Figure 12 shows the power spectra of six month running mean of $X$ in two cases of these uncoupled runs with a slowly varying forcing. The first one corresponds to a case with the annual cycle alone while the second one corresponds to a case with no annual cycle but a slowly varying forcing with period of four years in $G$. In both cases, it is evident that the system generates a considerable amount of low frequency signals with periods much longer than the forcing period. To understand how the slowly varying forcing introduces the low frequency response, we need to examine the nature of variations of the nonlinear system (1)-(3) for different values of the steady forcing $\left(F_{1}=G_{1}=0\right)$. In figure $13(\mathrm{a})$, we show some gross characters of the attractors (long term mean and standard deviation) of the system (1)-(3) with $G=1.0$ and for a range of values of $F$. The two symbols represent attractors attained from two different initial conditions. The system has periodic attractors for $F$ between 5.0 and 7.5 . For $F$ between 7.5 and 8.8 the system has a chaotic attractor. 

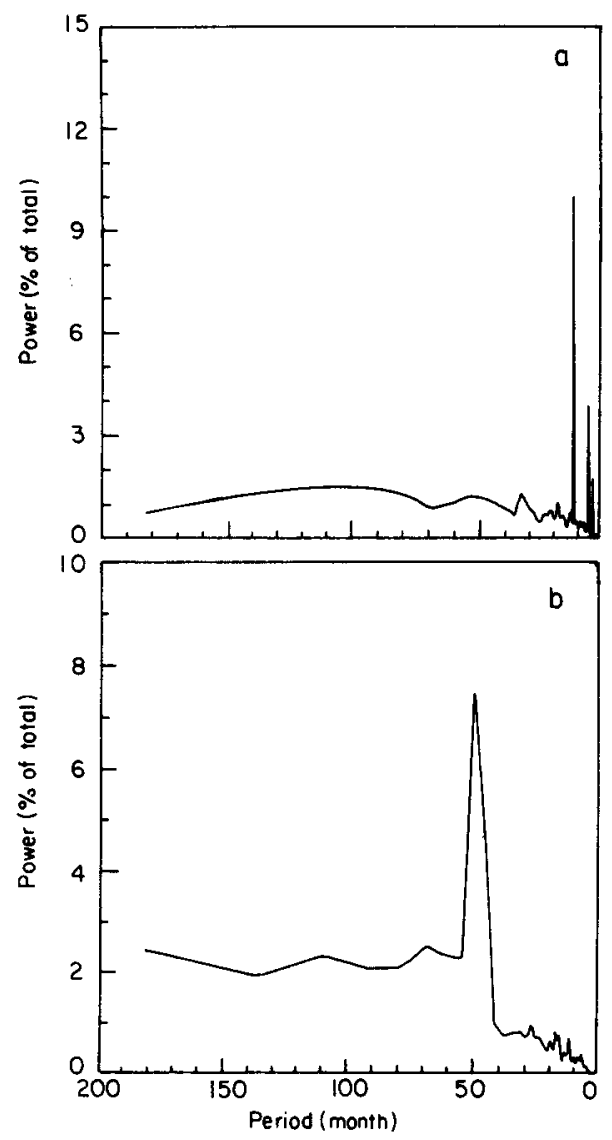

Figure 12. Spectra of six month running mean of $X$ with $\alpha=\beta=0$ and $C=1.0$ (a) with only the annual cycle in $F ; F_{0}=7.0, F_{1}=2.0, G_{0}=1 \cdot 0, G_{1}=0.0$ (b) with only a four year cycle in $G, F_{0}=8 \cdot 0 ; F_{1}=0.0, G_{0}=1 \cdot 0$ and $G_{1}=0.25$. Power spectra are calculated using 20 segments of $2^{14}$ days data in each case.

Beyond $F=8.8$ again the system goes to a periodic regime. We note that for $F$ between 5.0 and 7.0 , the system has at least two periodic orbits, one with high amplitude and low mean while the other with low amplitude and higher mean. Within the chaotic regime too, the system tends to have two preferred regimes, one with large amplitude oscillations (active regime) and another with small amplitude oscillations (weak regime). This is seen in figure $13(\mathrm{~b})$ where the mean and standard deviation calculated over three month segments over a period of 100 years is shown. The standard deviation shows the tendency for two preferred locations.

With this background, we may try to understand the response of the nonlinear system when the forcing changes periodically. When the forcing varies annually between two extremes, say between $F=9$ and $F=5$, the system reaches a chaotic attractor which consists of ${ }^{\circ}$ strong and a weak regime. The two regimes resemble the orbits of the multiple periodic attractors of constant forcings discussed earlier. Whether the system would tend to visit the weak or the strong regime depends on the previous history of states (or initial conditions, in a loose manner) which may favour one or the other regime. At many of these values of $F$, when they are held 

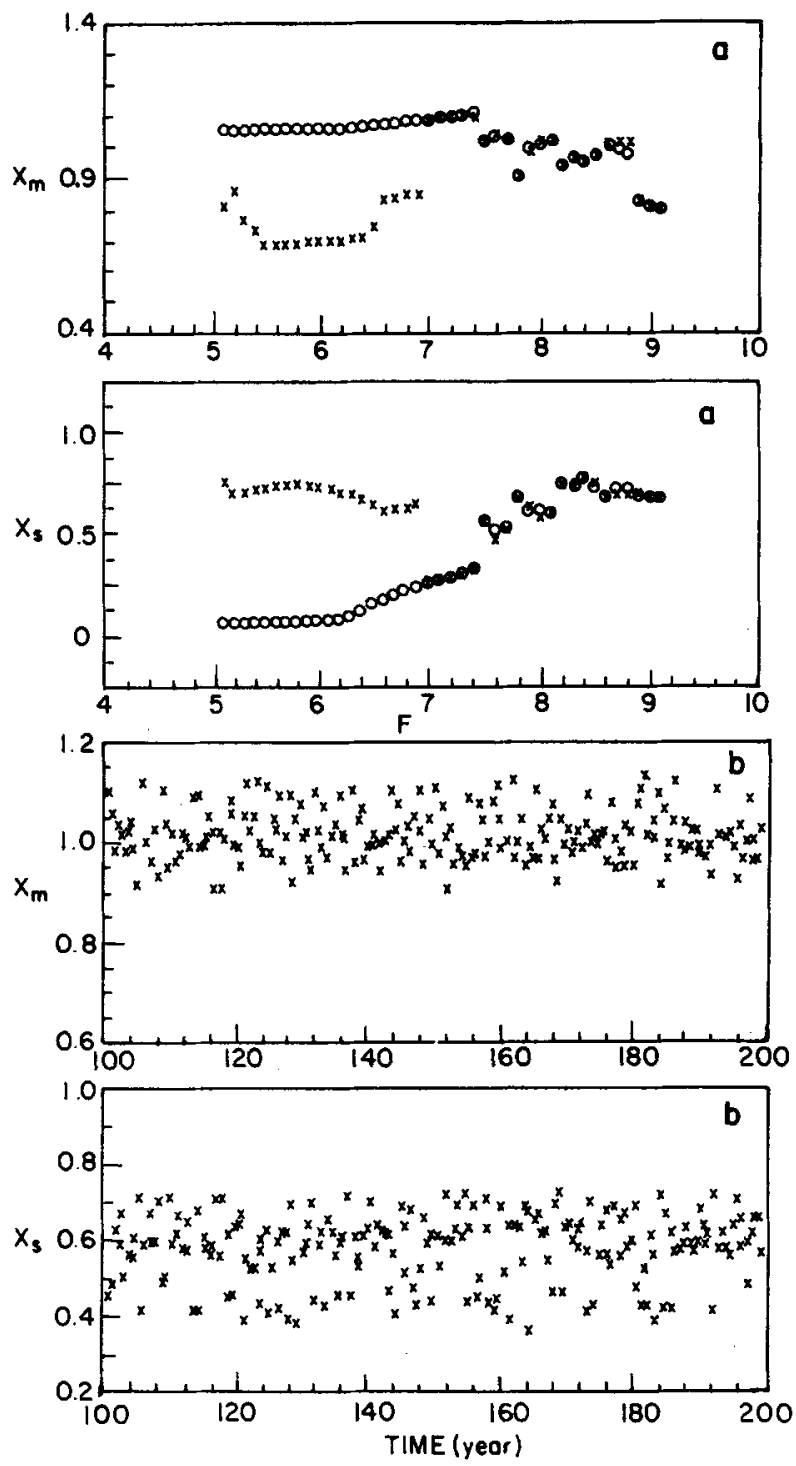

Figure 13. Some gross features of the attractors for $\alpha=\beta=0$ and $C=1.0$ with no annual cycle in $F$ and constant $G, G_{0}=1 \cdot 0, G_{1}=0.0, F_{1}=0.0$ (a) variation of long term mean $\left(X_{m}\right)$ and standard deviation $\left(X_{s}\right)$ for a range of values of $F(b)$ mean and standard deviation over 3 month segments for $F=8.0$.

constant, the system may possess nultiple periodic attractors which depend on the initial conditions. But when the forcing is varying, because of chaos, the system reaches a different state on the same day (or same value of $T$ ) of every year. This difference determines the difference in the subsequent behaviour of the systemwhether to visit the strong or the weak regime. This essentially is why the annual cycle itself is capable of introducing some inter-annual variability. Thus, the mechanism of generation of low frequency signals in the nonlinear system in our model is similar to the one discussed by Lorenz (1990). 

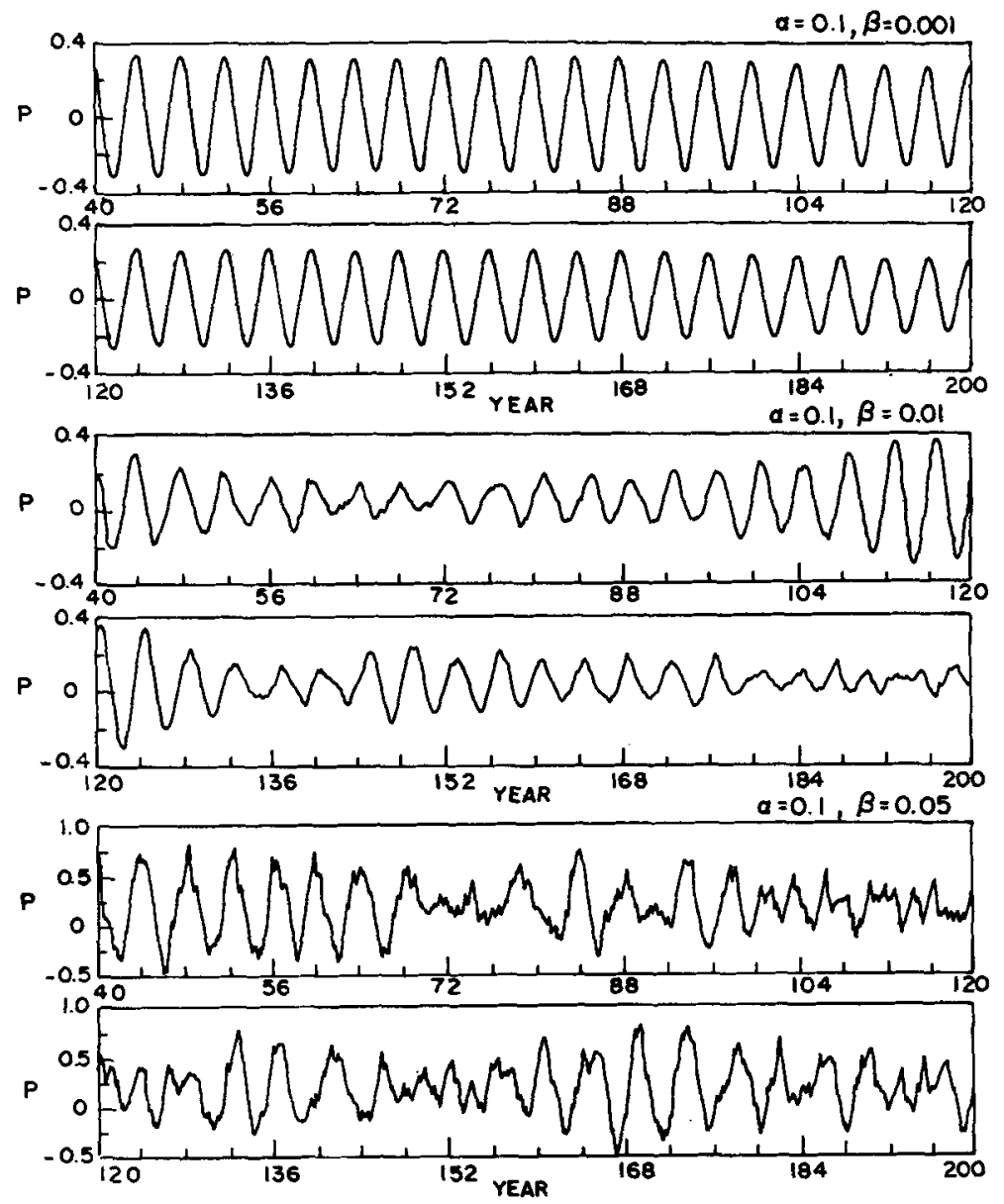

Figure 14. 160 year time series of $P$ for $\alpha=0.1$ and three increasing values of $\beta$ (a) $\beta=0.001$, (b) $\dot{\beta}=0.01$, (c) $\beta=0.05 . F$ and $G$ are the same as in figure 9 .

To understand how the interaction with the nonlinear high frequency component modifies the low frequency variability of the linear oscillator, a 160 year time series of $P$ for $\alpha=0.1$ for three increasing values of $\beta$ are shown in figure 14. For very small values of $\beta$, the linear oscillator remains nearly periodic while the amplitude is weakly modulated at a very low frequency. As the strength of $\beta$ is increased $(\beta=0 \cdot 01)$, the amplitude modulation becomes stronger. In other words, the spectrum broadens slightly around the four year period. With further increase of $\beta(\beta=0.05)$, the time series becomes quite aperiodic. Thus, increase in the coupling strength slowly broadens the spectrum of the low frequency oscillator and makes it aperiodic (figure 15). This broadening of the spectrum of the low frequency component with strength of coupling with the high frequency component is strikingly similar to the broadening of the spectrum of the ENSO signal in the coupled model of Cane and Zebiak with the increase in strength of convergence feedback in that model as discussed in section 2 of this article (ref. figure 4).

The experiments described in the previous paragraph for which $\alpha$ was 0.1 represents a case where the nonlinear high frequency component had a slowly varying forcings 


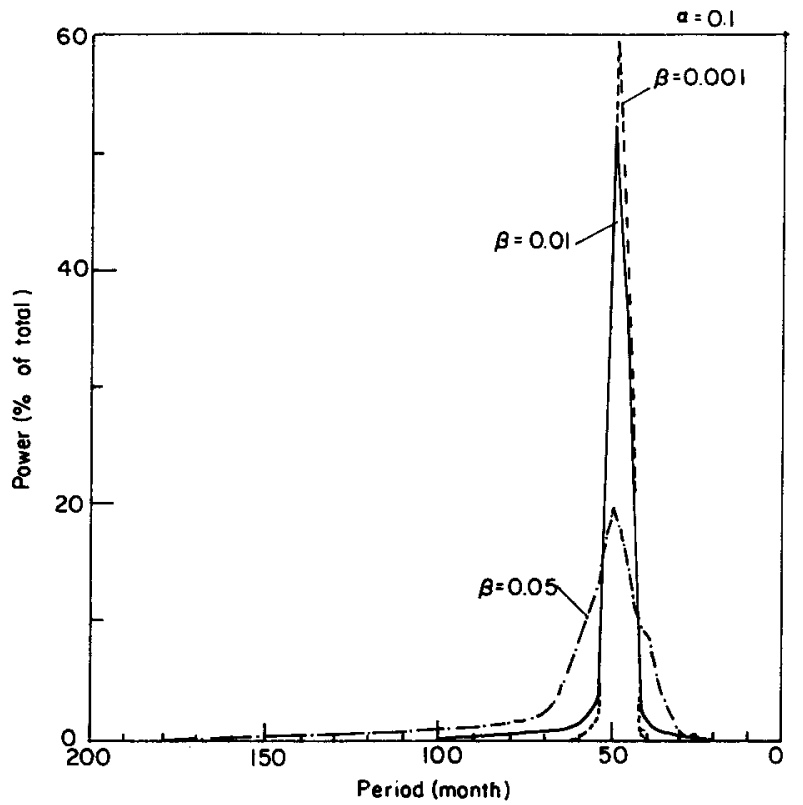

Figure 15. Spectra of $P$ for $\alpha=0.1$ and three increasing values of $\beta$.
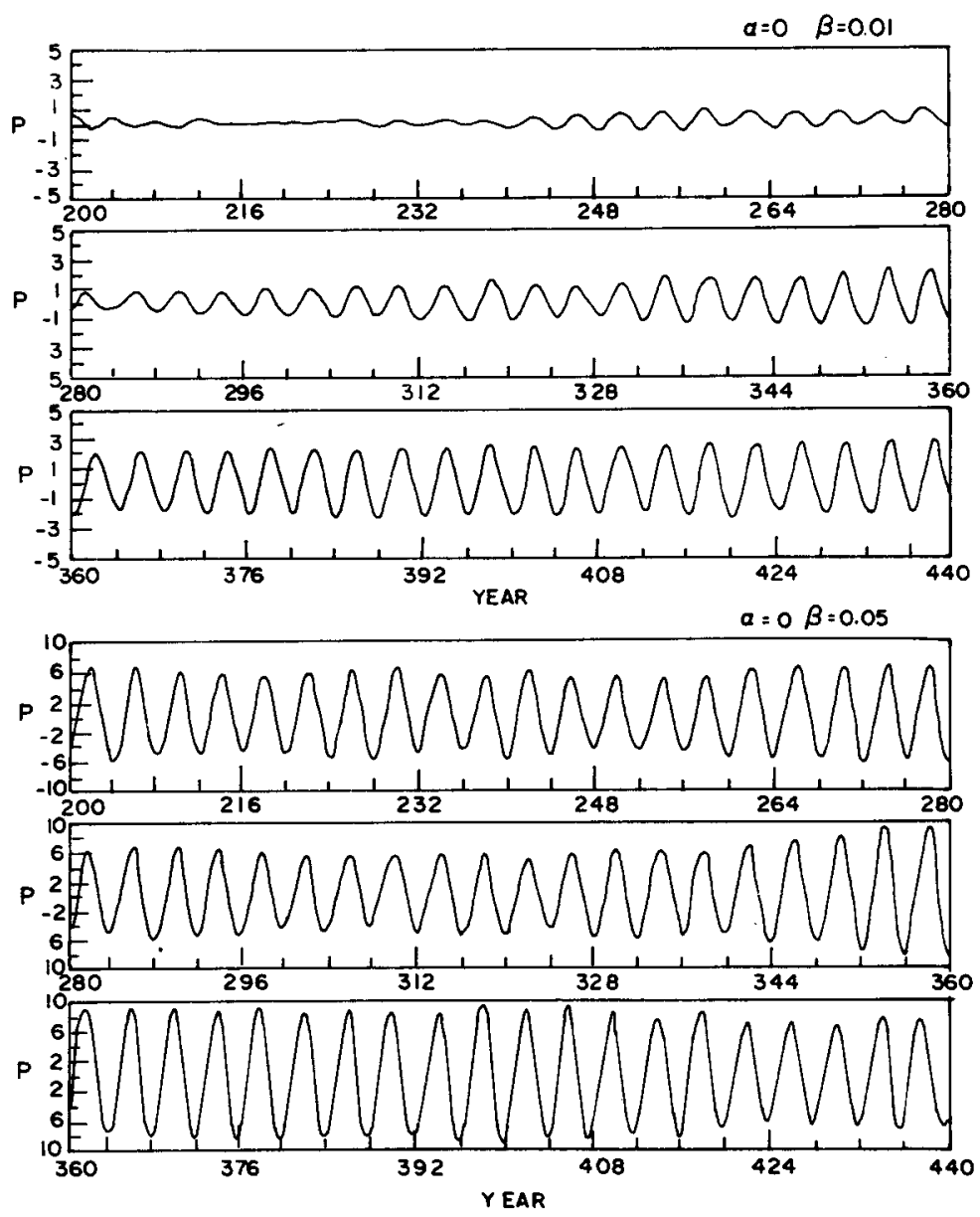

Figure 16. Time series of $P$ for $\alpha=0$ and two different values of $\beta$. (a) $\beta=0.01$, (b) $\beta=0 \cdot 1$. $F$ and $G$ are the same as in figure 9. 


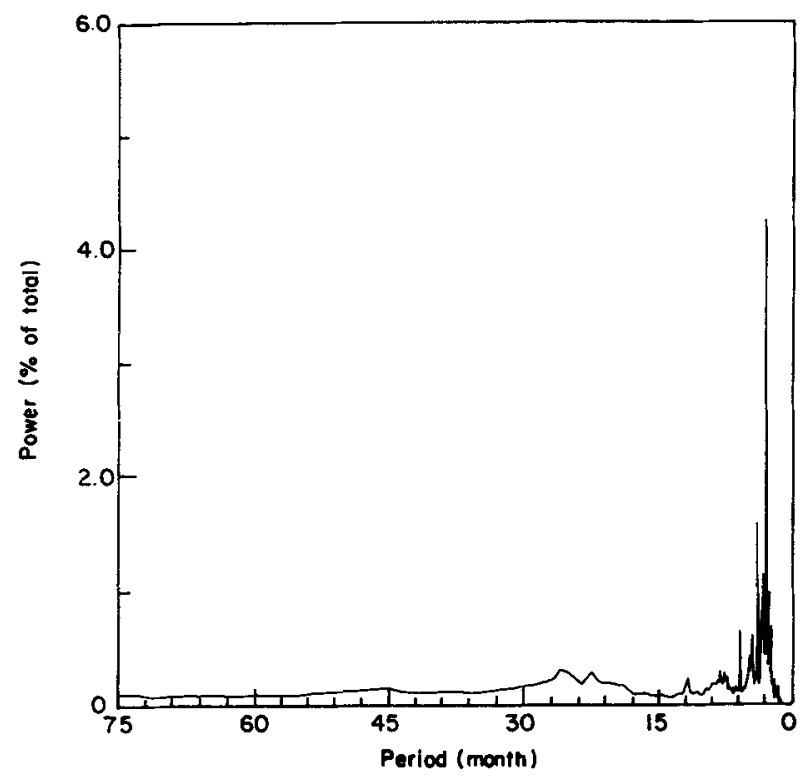

Figure 17. Power spectrum of uncoupled $X . F$ and $G$ are the same as in figure 9 .

with periods around four years. As a result, the nonlinear system generates significant amounts of low frequency signal as seen in figure $11(\mathrm{~b})$. We examined the role of these low frequency signals in the nonlinear system in producing the broadening of the spectrum of the low frequency linear oscillator by conducting some experiments with $\alpha=0$. Figure 16 shows a 240 year time series of $P$ for $\alpha=0$ and for two different values of $\beta$. It is seen that even for strong coupling strength $(\beta=0 \cdot 1)$, the variations in $P$ remain nearly periodic. The amplitude has a very slowly varying envelope. This amplitude envelope tends to grow very slowly in time. This seems to be due to the fact that the intrinsic spectrum of the nonlinear intra-seasonal oscillations without coupling to the low frequency oscillation, does not have much signal in the low frequency regime as shown in figure 17. Therefore, it appears that a low frequency component in the nonlinear system is necessary to produce aperiodicity in the low frequency oscillator. However, the low frequency variability in the nonlinear system is generated only through interaction with the low frequency oscillator. In other words, mutual coupling between the linear low frequency oscillator and the nonlinear intra-seasonal oscillations is necessary to produce aperiodicity in the linear low frequency oscillator. A brief report of the work presented in this section is being published elsewhere (Krishnamurthy et al 1993).

\section{Conclusions}

The predictability of the tropical short term climate is limited by the aperiodicity of the low frequency variability of the coupled ocean-atmosphere system. An attempt has been made in this article to gain insight into the mechanism leading to the aperiodicity of the low frequency variability of the coupled system.

An examination of the observed inter-annual variability in the tropics as well as simulations of some coupled models (Cane and Zebiak 1985; Zebiak and Cane 1987) indicates that the variability is aperiodic but the spectrum has a dominant peak with 
a period around four years. The oscillations associated with the dominant low frequency mode have very large horizontal scales $(6-7$ thousand $\mathrm{km})$. Such a planetary scale phenomenon is expected to behave nearly linearly and is unlikely to produce the broadening of the spectrum by itself. Thus, in order to produce aperiodicity of the low frequency variability, the dominant low frequency mode must coexist with other unstable normal modes of the coupled system.

Earlier stability analyses of the coupled system (Hirst 1986, 1988) showed the existence of only one low frequency mode. Other unstable modes of the coupled system, if they exist, must be related to physical processes not taken into account in these early stability analyses. To have an idea about such physical processes that may lead to new unstable modes in the coupled system, a number of sensitivity studies were carried out with the $\mathrm{CZ}$ model. These studies showed that for the standard set of parameters used by $\mathrm{CZ}$, the convergence feedback contributes significantly to the aperiodicity of the model. In the absence of convergence feedback, the model variability is perfectly periodic. With the increase of the strength of the convergence feedback, model variability goes from a line spectrum to a broad band spectrum. A linear stability analysis was then carried out to investigate if the convergence feedback could introduce some new unstable modes. This analysis reveals that, the convergence feedback indeed introduces a set of higher frequency unstable intra-seasonal modes. In the long wavelength limit, the growth rate of these modes is weaker than that of the low frequency mode. Therefore, in the longwave limit, the low frequency mode is dominant. However, on the shorter wavelength side, the low frequency mode is stable but the high frequency modes have considerable growth rate. Thus, in the shorter wavelength regime, the higher frequency intra-seasonal modes are expected to dominate. Therefore, in the coupled system we expect a low frequency long wave mode to coexist with a number of higher frequency shorter wave intra-seasonal modes. Since, the scales of the intra-seasonal modes are much shorter, it is expected that the nonlinear interaction between them would be important.

We then propose that the aperiodicity in the low frequency variability in the tropics results from an interaction between a linear oscillator corresponding to the dominant four year low frequency mode and the nonlinear intra-seasonal modes of the coupled system. We demonstrate this by representing the nonlinear interactions between the intra-seasonal modes by a prototype low order nonlinear dynamical system and coupling it to a linear oscillator with a period of four years. We show that if the coupling is only one way, that is if the intra-seasonal component acts as a forcing to the low frequency linear oscillator but does not get affected by the linear oscillator, it cannot broaden the spectrum of the low frequency oscillator. However, if the coupling were both ways, interaction with the nonlinear intra-seasonal component results in broadening of the low frequency spectrum. The low frequency oscillator behaves like a slowly varying forcing to the nonlinear intra-seasonal oscillations. This gives rise to low frequency components to the intra-seasonal oscillations. The existence of such a low frequency tail in the nonlinear higher frequency oscillations seems to be crucial for producing the aperiodicity in the low frequency oscillator. It is rather intriguing that our conceptual model could simulate many features of real coupled model simulations such as that with the $\mathrm{CZ}$ model.

In Goswami and Shukla (1991), the existence of two time scales for the predictability of the tropical coupled system was discovered. However, the physical processes responsible for the two time scales were not clear from that study. The results presented 
in this study may throw some light regarding the two time scales in the predictability of the tropical coupled system. The slow time scale is expected to be related to the low frequency oscillator while the fast time scale is associated with the broadening of the low frequency spectrum. However the broadening of the low frequency results from interactions with the nonlinear intra-seasonal oscillations. Therefore, the fast time scale is essentially related to the nonlinearities associated with the higher frequency intra-seasonal oscillations. If the saturation level of the errors associated with the higher frequency oscillations is lower than the saturation level of the errors associated with the low frequency oscillation, we may be able to see the two time scales in the error growth of the coupled system. We are currently examining the predictability of the coupled system (1)-(5) using a large ensemble of identical twin experiments.

\section{References}

Barnett T P, Graham N, Cane M A, Zebiak S E, O'Brien J and Legler D 1988 On the prediction of the El-Nino of 1986-87; Science 241 192-196

Battisti D S 1988 Dynamics and thermodynamics of a warm event in a coupled tropical atmosphere-ocean model; J. Atmos. Sci. 45 2889-2919

Battisti D S and Hirst A C 1989 Interannual variability in the tropical atmosphere/ocean system: Influence of the basic state and ocean geometry; J. Atmos. Sci. 46 1687-1712

Blumenthal M B 1991 Predictability of a coupled ocean-atmosphere model; J. Climate 4766-784

Cane M A and Zebiak S E 1985 A theory for El-Nino and the Southern Oscillation; Science 228 1085-1087

Cane M A, Zebiak S E and Dolan S C 1986 Experimental forecasts of the 1982/83 El-Nino; Nature 321 827-832

Charney J G and Shukla J 1981 Predictability of monsoons. In Monsoon Dynamics. (ed.) Sir James Lighthill and R P Pearce (Cambridge: University Press) p. 99-110

Gill A E 1980 Some simple solutions of heat induced tropical circulation; Q. J. R. Meteorol. Soc. 106 447-462

Goswami B N and Selvarajan S 1991 Convergence feedback and unstable low frequency oscillations in a simple coupled ocean-atmosphere model; Geophys. Res. Lett. 18 991-994

Goswami B N and Shukla J 1991 Predictability of a coupled ocean-atmosphere model; J. Climate 43-22

Goswami B N and Shukla J 1993 Aperiodic variability in the Cane-Zebiak model: A diagnostic study; $J$. Climate 6 (in press)

Hirst A C 1986 Unstable and damped equatorial modes in simple coupled ocean-atmosphere models; $J$. Atmos. Sci. 43 606-630

Hirst A C 1988 Slow instabilities in tropical ocean basin-global atmosphere models; J. Atmos. Sci. 45 $830-852$

Hirst A C and Lau K M 1990 Intraseasonal and interannual oscillations in coupled ocean-atmosphere models; $J$. Climate 3 713-725

Krishnamurthy V, Goswami B N and Legnani 1993 A conceptual model for the aperiodicity of interannual variability in the tropics. Geophys. Res. Lett. 20 (in press)

Latif $\mathrm{M}$ and Flugel 1991 An investigation of short-range climate predictability in the tropical Pacific; $J$. Geophys. Res. 96 2661-2673

Lau K M and Shen S 1988 On the dynamics of intraseasonal oscillations and ENSO; J. Atmos. Sci. 45 1781-1797

Lorenz E N 1984 Irregularity a fundamental property of the atmosphere; Tellus A36 98-110

Lorenz E N 1990 Can chaos and intransitivity lead to interannual variability?; Tellus A42 378-389

Philander S G H 1990 El Nino, La Nina and Southern Oscillation (Academic Press) pp 289

Philander S G H, Pacanowski R C and Yamagata T 1984 Unstable air sea interactions in the tropics; $J$. Atmos. Sci. 41 604-613

Philander S G H, Pacanowski R C, Lau N C and Nath M J 1992 A simulation of the Southern Oscillation. Part 2: Results from high resolution coupled general circulation models of the ocean and atmosphere; J. Climate 5 308-329 
Rasmusson E M and Carpenter T H 1982 Variations in tropical sea surface temperature and surface wind fields associated with the Southern Oscillation/El Nino; Mon. Weather Rev. 110 354-384

Schopf P S and Suarez M J 1988 Vascillations in a coupled ocean-atmosphere model; J. Atmos. Sci. 45 $549-566$

Selvarajan S and Goswami B N 1992 On the stability of a coupled ocean atmosphere system in the presence of wave-CISK; Proc. Indian Acad. Sci. (Earth Planet. Sci.) 101 153-176

Suarez M J and Schopf P S 1988 A delayed oscillator for ENSO; J. Atmos. Sci. 46 3283-3287

Shukla J 1981 Dynamical predictability of monthly means; J. Atmos. Sci. 38 2547-2572

Shukla J 1985 Predictability in advance geophysics (Academic Press) B28 pp. 87-122

Shukla $J$ and Wallace J M 1983 Numerical simulation of the atmospheric response to equatorial Pacific sea surface temperature anomalies; J. Atmos. Sci. 40 1613-1630

Zebiak S E and Cane M A 1987 A model El-Nino/Southern Oscillation; Mon. Weather Rev. 115 2262-2278 